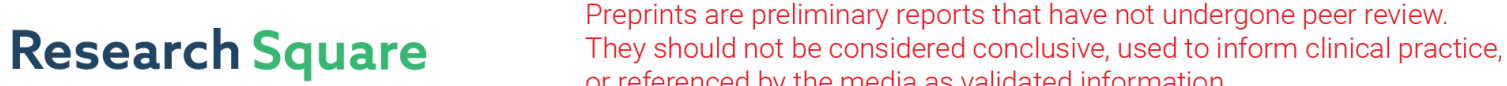 or referenced by the media as validated information. \\ Transmission type Dual-frequency Vortex Beams Generator for Multiple OAM Modes
}

\section{Zhang}

China Jiliang University

\section{J. Li ( $\nabla$ forever-li@126.com )}

China Jiliang University

\section{Research Article}

Keywords: terahertz wave , transmission-type metasurface, convolution operation OAM

Posted Date: July 9th, 2021

DOI: https://doi.org/10.21203/rs.3.rs-629672/v1

License: (c) (i) This work is licensed under a Creative Commons Attribution 4.0 International License. Read Full License 


\title{
Transmission type dual-frequency vortex beams generator for multiple OAM modes
}

\author{
Zhang Lei, Li Jiu-sheng* \\ Centre for THz Research, China Jiliang University, Hangzhou 310018, China
}

\begin{abstract}
We design a transmission-type metasurface to generate terahertz dual-frequency orbital angular momentum $(\mathrm{OAM})$ vortex beams with different topological charges $(l=1, l=2)$ at frequency of $0.81 \mathrm{THz}$ and $1.63 \mathrm{THz}$. By adding a deflection factor, the proposed metasurface produces a OAM vortex beam with a preset tilted direction at two frequencies. By utilizing a complex convolution operation, we can achieve two symmetric tilted beams with different topological charges $(l=1, l=-1)$ at two frequencies on the same metasurface. These OAM vortex beams can effectively improve communication system capacity, and have potential applications in terahertz communication.
\end{abstract}

Keywords: terahertz wave $\cdot$ transmission-type metasurface $\cdot$ convolution operation OAM

\section{Introduction}

OAM beams with $\exp (i l \varphi)$ phase factor ( $\varphi$ is azimuth angle and $l$ is topological charge) can increase information capacity infinitely in theory. The topological charge $l$ can take any integer values, and OAM beams with different integer orders are orthogonal to each other (Gibson et al. 2004; Chen et al. 2011). In 1992, OAM was firstly proposed in optical region (Allen at el. 1992), and then the concept of photon OAM was extended to microwave and terahertz wave regime (Tang at el. 2019; Zhang et al. 2020; Zhang et al. 2017; Akram et al. 2019; Qin et al. 2018; Li et al. 2016). Especially, terahertz technology has been designated as 6G communication band in 2019 (Yang et al. 2020; Qi et al. 2020), terahertz OAM vortex beams a hot research issues for efficient increasing $6 \mathrm{G}$ wireless communication capacity. Due to its small in size and easy to integrate with other devices, metasurface-based OAM beam generators has attracted great interests in the past few years (Tang et al. 2019; Fan et al. 2020; Xie et al. 2019; Ding et al. 2019; Ran et al. 2018; Wang et al. 2018). However, most of the reported dual-frequency vortex beam generators are based on reflective metasurfaces(Ji et al. 2019; Xin et al. 2020; Wang et al. 2019), there are few articles about transmissive dual-frequency vortex beam generator. Therefore, it is of great significance for study a transmission-type OAM generator at different frequency bands.

In this paper, we propose a transmissive metasurface which can generate terahertz vortex beams at two frequencies. Firstly, the vortex beams generator produces the cross-polarized OAM vortex beams without deflection angle at 0.81 $\mathrm{THz}$ and $1.63 \mathrm{THz}$, respectively. The OAM mode purity is larger than 70\%. Secondly, by adding a deflection factor, the proposed transmissive metasurface generate a tilted dual-frequency OAM vortex beams with deflection angle $28^{\circ}$ and $13^{\circ}$ at $0.81 \mathrm{THz}$ and $1.63 \mathrm{THz}$, respectively. Finally, by using complex convolution operation, the proposed metasurface achieves two symmetric tilted vortex beams at two same frequencies with different topological charges $(l=+1$ and $l=-1)$ towards the directions of $+x$ and $-x$, respectively. The simulation results show that such a metasurface has the potential applications in future $6 \mathrm{G}$ communication.

\section{Structure design and discussion}

Figure 1 describes the schematic diagram of the proposed dual-frequency OAM generator under circularly polarized terahertz wave incidence. Figure 1(a) depicts the generation of the cross-polarized OAM vortex beams in normal direction at two diffident frequencies under a circularly polarized terahertz wave incidence. In Fig.1(b), the transmissive cross-polarized beams have different deflection angle according to a preset direction at the two selected frequencies. Figure 1(c) shows the transmission terahertz wave having two symmetric tilted vortex beams with different topological charges at two diffident frequencies. The vortex beams deflection angle at $f_{1}$ is differ from that of $f_{2}$. Figure 2 displays the unit cell of the proposed dual-frequency vortex beams generator, which is composed of a top metallic layer and the same size bottom metallic layer separated by polyimide (with relative permittivity of 3.5 and a thickness of $20 \mu \mathrm{m}$ ). We used the full wave simulation software CST to optimize and obtain the geometric parameters as follows: $\mathrm{w}=10 \mu \mathrm{m}, \mathrm{g}=13 \mu \mathrm{m}, \mathrm{r}=15 \mu \mathrm{m}, \mathrm{d}=35 \mu \mathrm{m}, \mathrm{t}_{1}=0.2 \mu \mathrm{m}, \mathrm{t}_{2}=20 \mu \mathrm{m}$ and the period of the unit cell is set to $100 \mu \mathrm{m}$.

\footnotetext{
* Li Jiu-sheng

forever-li@126.com

Centre for THz Research, China Jiliang University, Hangzhou 310018, China
} 


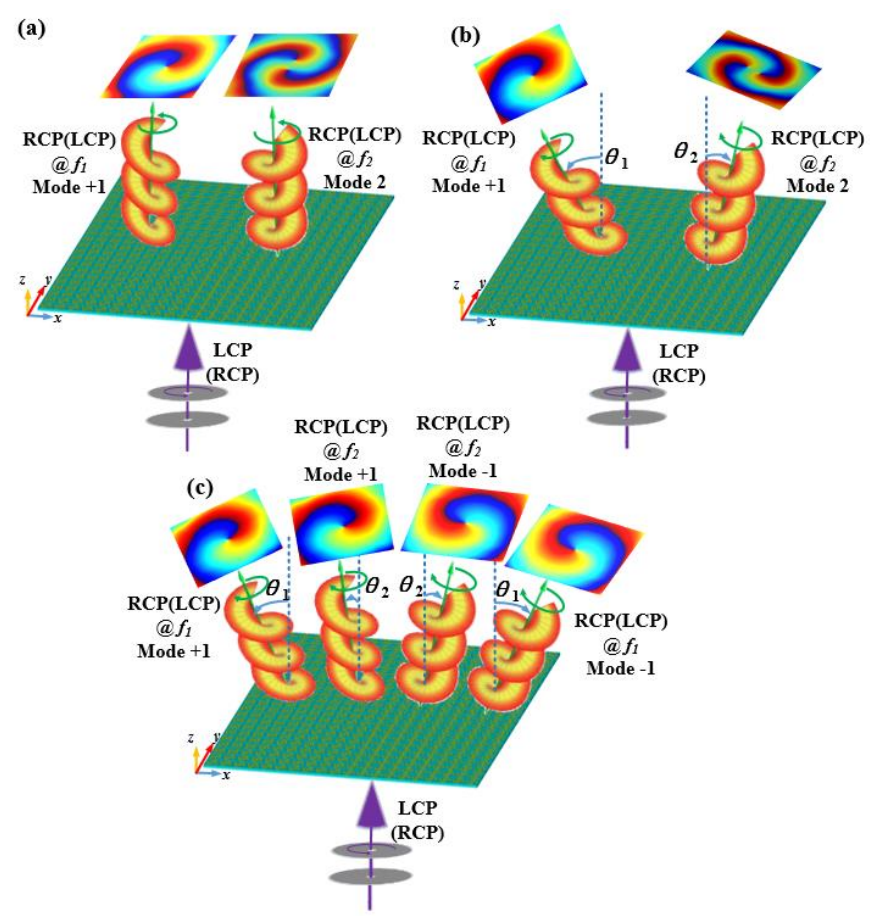

Fig 1. Schematic illustration of the transmissive cross-polarized OAM generator (a) The transmission terahertz wave propagates in two vertical directions, (b) in tilted beam directions with different deflection angle at two frequencies, (c) in symmetric vortex beams with different deflection angle at two frequencies under circularly polarized (CP) terahertz wave incidence.
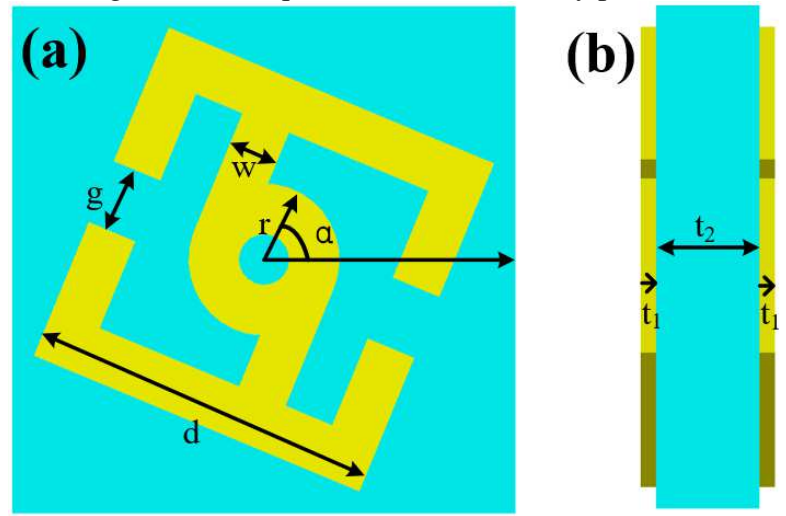

Fig 2. Geometric parameters of unit cell (a) top view, (b) side view.

According to the phase principle of Pancharatnam-Berry, when a plane wave vertically illuminates on the unit cell, the structure can change the beam propagation direction with the rotation angle $(\alpha)$. The rotation matrix can be described as

$$
R_{\alpha}=\left(\begin{array}{cc}
\cos (\alpha) & \sin (\alpha) \\
-\sin (\alpha) & \cos (\alpha)
\end{array}\right)
$$

The matrix of the transmission field is obtained:

$$
T_{\text {new }}=R_{\alpha}^{-1} T R_{\alpha}
$$

The incident circularly polarized wave can be expressed as

$$
E_{i}=E_{0}(1 \pm \mathrm{i})^{\mathrm{T}}
$$

"+ (-)" means the incidence of left-handed (right-handed) circularly polarized wave (LCP/RCP). Thus, the matrix of the transmission field can be calculated by

$$
E_{t}=T_{n e w} \cdot E_{i}=\frac{1}{2} \cdot \mathrm{E}_{0} \cdot\left(T_{x x}+T_{y y} \pm \mathrm{i} T_{x y} \mp \mathrm{i} T_{y x}\right)\left(\begin{array}{c}
1 \\
\pm \mathrm{i}
\end{array}\right)+\frac{1}{2} \cdot \mathrm{E}_{0} \cdot\left(T_{x x}-T_{y y} \pm \mathrm{i} T_{x y} \pm \mathrm{i} T_{y x}\right) \cdot e^{ \pm \mathrm{i} 2 \alpha}\left(\begin{array}{l}
1 \\
\mp \mathrm{i}
\end{array}\right)
$$

As seen from Eq. (4), the transmission field contains co-polarized wave and cross-polarized wave. Obviously, only the cross-polarized wave introduces an additional phase factor related to OAM. The additional phase factor equals $2 \alpha$ when the unit cell rotates an angle of $\alpha$. Figure 3(a) clearly demonstrates the transmission coefficient of the transmitted beams. One can see that the cross-polarized transmission amplitudes are greater than 0.8 at the frequency of $0.81 \mathrm{THz}$ 
and 1.63 THz. Moreover, the transmission coefficient is not varied as the change of the rotation angle. As can be seen from Fig.3(b), the phase of the transmitted beam change $2 \alpha$ with the increase rotation angle $\alpha$ of the unit cell. Thus, the required phase can be obtained through different rotation angles of the unit cell.
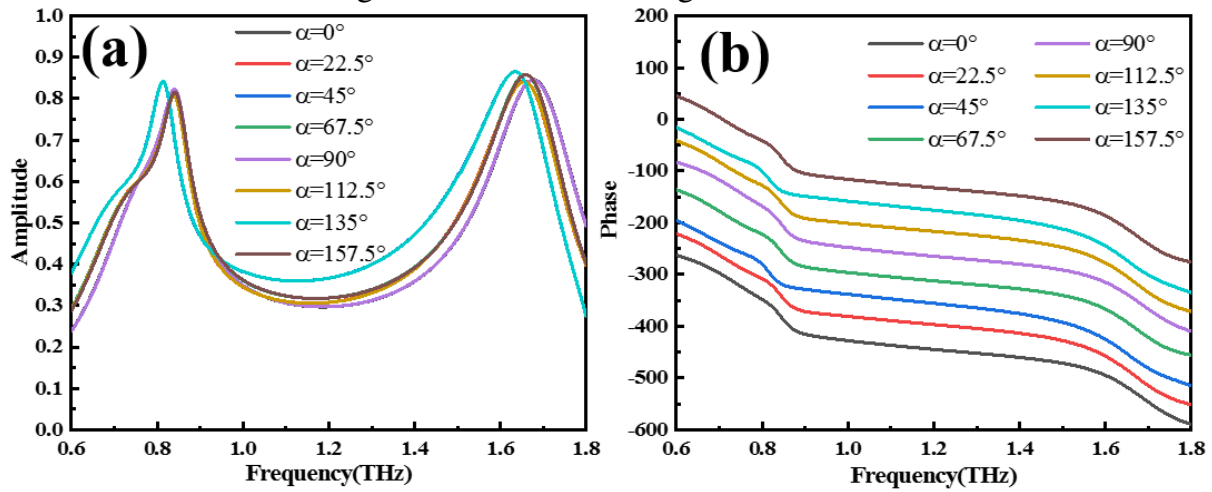

Fig 3. (a) Transmission amplitude, (b) phase with different corner under the LCP (RCP)

\subsection{Dual-frequency transmissive vortex beams generator without deflection angle}

Firstly, according to the Pancharatnam-Berry phase principle, we design the metasurface can generate terahertz dualfrequency vortex beams without deflection angle. In order to satisfy the phase of the vortex beam with topological charges $(l)$, the phase distribution for each position $(x, y)$ can be given by (Fan and Cheng 2020)

$$
\varphi_{1}(x, y)=l \cdot \tan ^{-1}(y / x)
$$

where $l$ is the topological charge. In order to simplify the design, the metasurface can be divided into $N$ triangular regions, and the phase distribution of each region can be calculation by

$$
\varphi_{1}(x, y)=\frac{2 \pi}{N}\left[\frac{l \cdot \tan ^{-1}(y / x)}{2 \pi / N}+1\right]
$$

where $N$ is the total number of the metasurface regions. Here, the metasurface pattern is divided as eight regions (i.e. $N=8)$.

Therefore, the phase is twice as much the rotation angle of the unit cell. The proposed metasurface (Named as $\mathrm{M}_{1}$ and $\mathrm{M}_{2}$ ) phase distributions for OAM vortex beams with $l=1$ and $l=2$ are presented in Fig. 4 . The metasurface consists of $24 \times 24$ unit-cells $(2400 \mu \mathrm{m} \times 2400 \mu \mathrm{m})$ with the different values of topological charge.
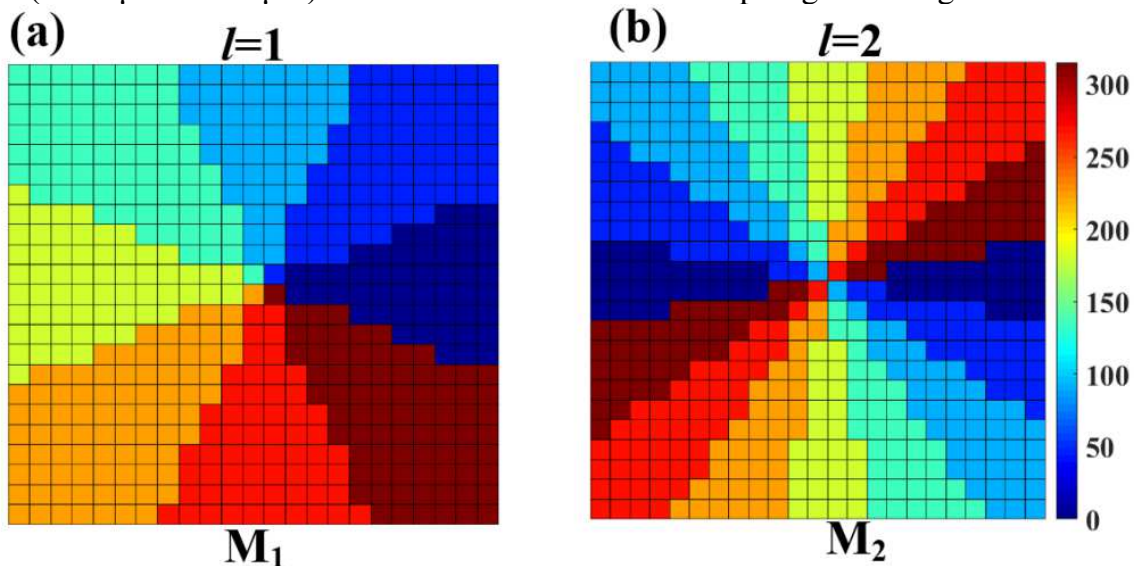

Fig 4. The phase distribution of the vortex beam generator with topological charges (a) $l=1$ and (b) $l=2$.

The proposed metasurfaces are simulated and analyzed by using CST, an LCP (RCP) Gaussian beam is set to perpendicularly incident to the proposed metasurfaces, the amplitude of the electric field in the $x$ and $y$ directions are set as $1 \mathrm{~V} / \mathrm{m}$. Figure 5 record the near-field phase and amplitude of the cross-polarized transmitted beam electric field. When an LCP (RCP) Gaussian beam perpendicularly incident on metasurface, it can be observed from Fig.5(a) and Fig.5(b) that the phase and amplitude distribution at $0.81 \mathrm{THz}$ and $1.63 \mathrm{THz}$ of $l=1$, respectively. The phase and amplitude distribution of $l=2$ are displayed in Fig. 5(c) and Fig. 5(d), respectively. The simulation results are consistent with the characteristics of the vortex beam. 

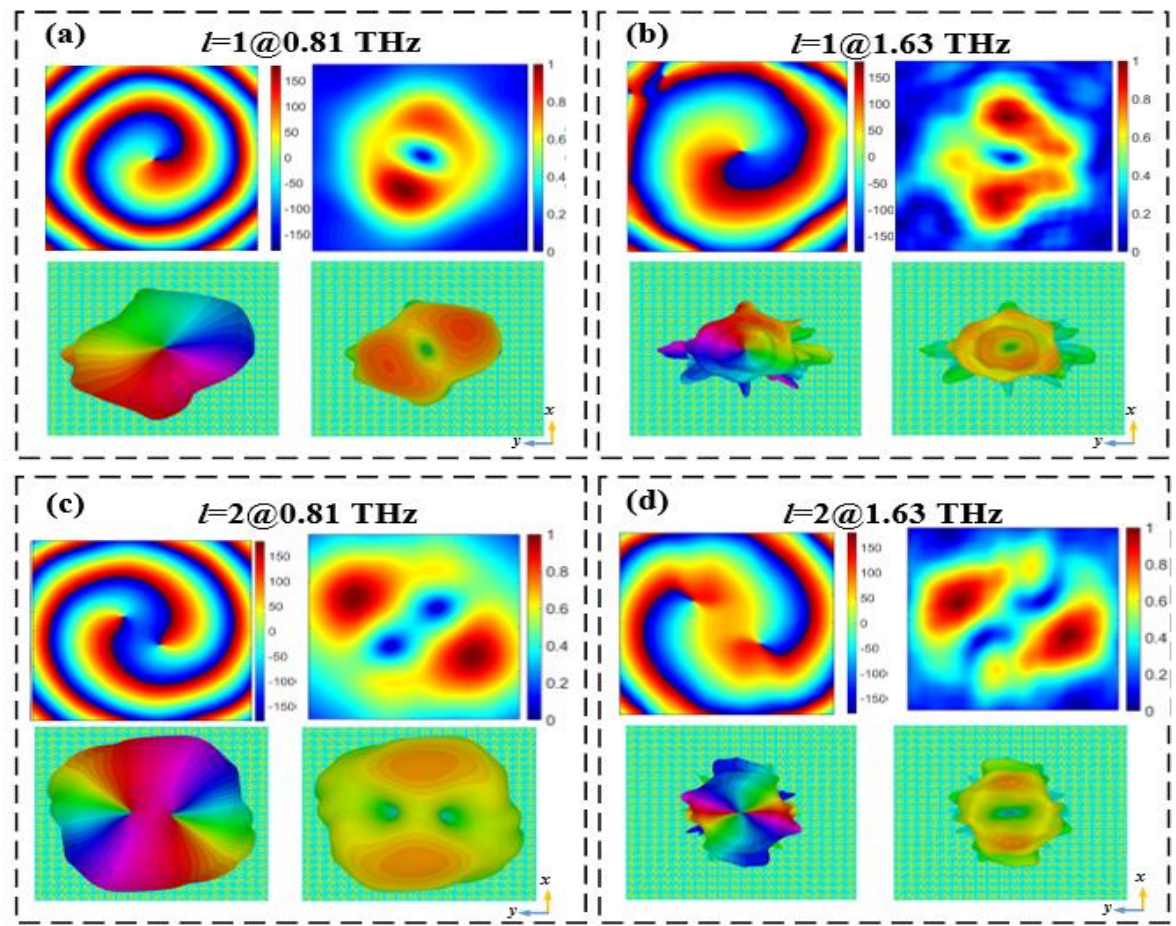

Fig 5. Phase and amplitude of cross-polarized transmissive beam (a) $l=1$ at $0.81 \mathrm{THz}$ (b) $l=1$ at $1.63 \mathrm{THz}$ (c) $l=2$ at $0.81 \mathrm{THz}(\mathrm{d})$ $l=2$ at $1.63 \mathrm{THz}$.

In order to evaluate the efficiency of the vortex beams generator, we calculate the mode purity of OAM vortex beams with different topological charges based on the Fourier transform. The Fourier relationship can be given by

$$
\left\{\begin{array}{c}
\alpha(\varphi)=\sum_{l=-\infty}^{+\infty} A_{l} \cdot \exp (\mathrm{i} l \varphi) \\
A_{l}=\frac{1}{2 \pi} \int_{-\pi}^{\pi} d \varphi \alpha(\varphi) \cdot \exp (\mathrm{i} l \varphi)
\end{array}\right.
$$

where $\alpha(\varphi)$ is the phase and $\exp (\mathrm{i} / \varphi)$ is the vortex harmonic. The proportion of the OAM mode purity at $0.81 \mathrm{THz}$ and $1.63 \mathrm{THz}$ are presented in Fig. 6. From the figure, one can clearly see that, the mode purity of OAM vortex beams is about $71.7 \%$ at $0.81 \mathrm{THz}$ and $70.7 \%$ at $1.63 \mathrm{THz}$ for topological charge $l=+1$. In addition, the mode purity equals $79.1 \%$ at $0.81 \mathrm{THz}$ and $81.9 \%$ at $1.63 \mathrm{THz}$ as the topological charge is $l=2$.
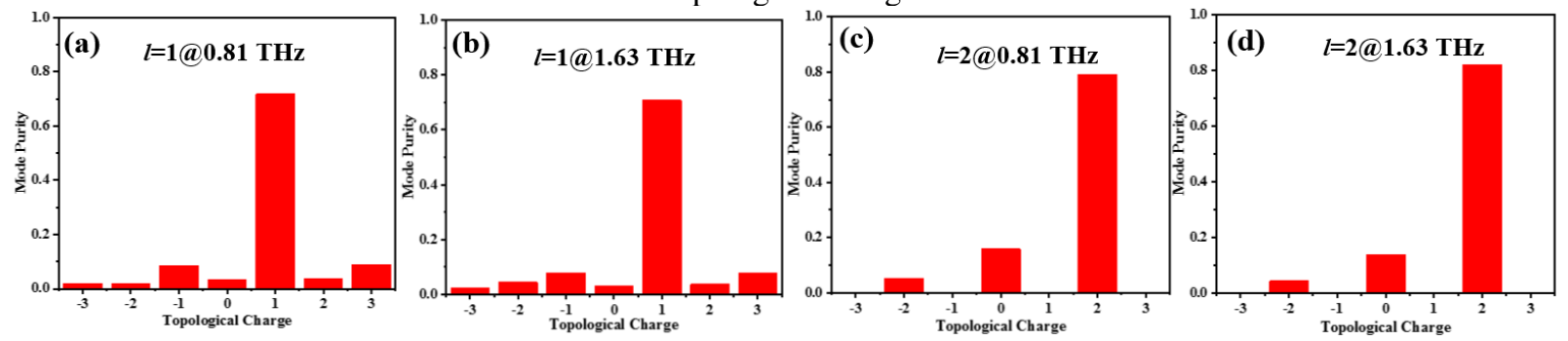

Fig 6. Mode purity of the vortex beam for (a) $l=1$ at $0.81 \mathrm{THz}$

(b) $l=1$ at $1.63 \mathrm{THz}$ (c) $l=2$ at $0.81 \mathrm{THz}$ (d) $l=2$ at $1.63 \mathrm{THz}$.

\subsection{Dual-frequency transmissive vortex beams generator with two deflection angles}

Secondly, the proposed metasurface can produce dual-frequency tilted OAM vortex beams by adding a deflection factor. The vortex beams carry a deflection angle along $x$ direction by adding the OAM pattern to the gradient phase, and the final phase distribution can be written as (Xin at al. 2020)

where $\theta$ is the deflection angle can be calculated by

$$
\varphi_{2}(x, y)=\varphi_{1}(x, y)+(2 \pi \cdot \sin \theta) / \lambda
$$

$$
\theta=\sin ^{-1}(\lambda / \tau)
$$

where $\tau$ is the period of the gradient coding sequence. Here, $\tau=8 \mathrm{P}=800 \mu \mathrm{m}$, the deviation angle $\theta$ can be calculated as $27.5^{\circ}$ at $0.81 \mathrm{THz}$ and $13^{\circ}$ at $1.63 \mathrm{THz}$, respectively. 


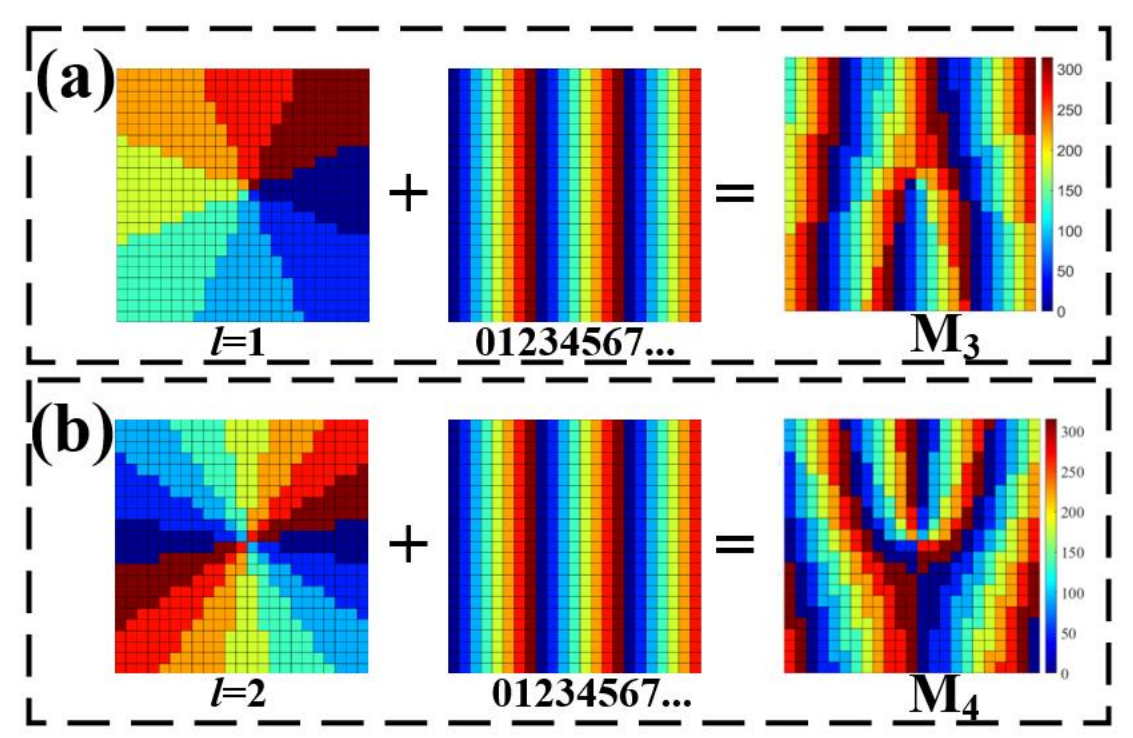

Fig 7. The calculation process and the final total phase distribution for (a) $l=1$ and (b) $l=2$.

As presented in Fig. 7(a), we add the OAM pattern with $l=1$ to the gradient phase sequence $(01234567 \ldots)$ with a deflection angle along $x$ direction, and the total phase distribution (Named $\left.\mathrm{M}_{3}\right)$ can be obtained. Likewise, we add the same gradient phase with a deflection angle to OAM pattern with $l=2$, and the total phase distribution $\left(\mathrm{Named}_{4}\right)$ can be achieved, as plotted in Fig.7(b). The proposed metasurfaces, which consists of $24 \times 24(2400 \mu \mathrm{m} \times 2400 \mu \mathrm{m})$ unit-cells with $l=1(l=2)$, is illuminated vertically by an LCP (RCP) Gaussian beam. Figure 8 depicts the 2D far-field amplitude patterns, the phase and amplitude distribution at different operating frequencies. It can be clearly seen that the phase and amplitude distribution are in good agreements with the characteristic of vortex beams. The $2 \mathrm{D}$ far-field amplitude patterns shows deviation angle is $30^{\circ}$ at $0.81 \mathrm{THz}$ and $15^{\circ}$ at $1.63 \mathrm{THz}$, respectively, which consistent with previous theoretical prediction.

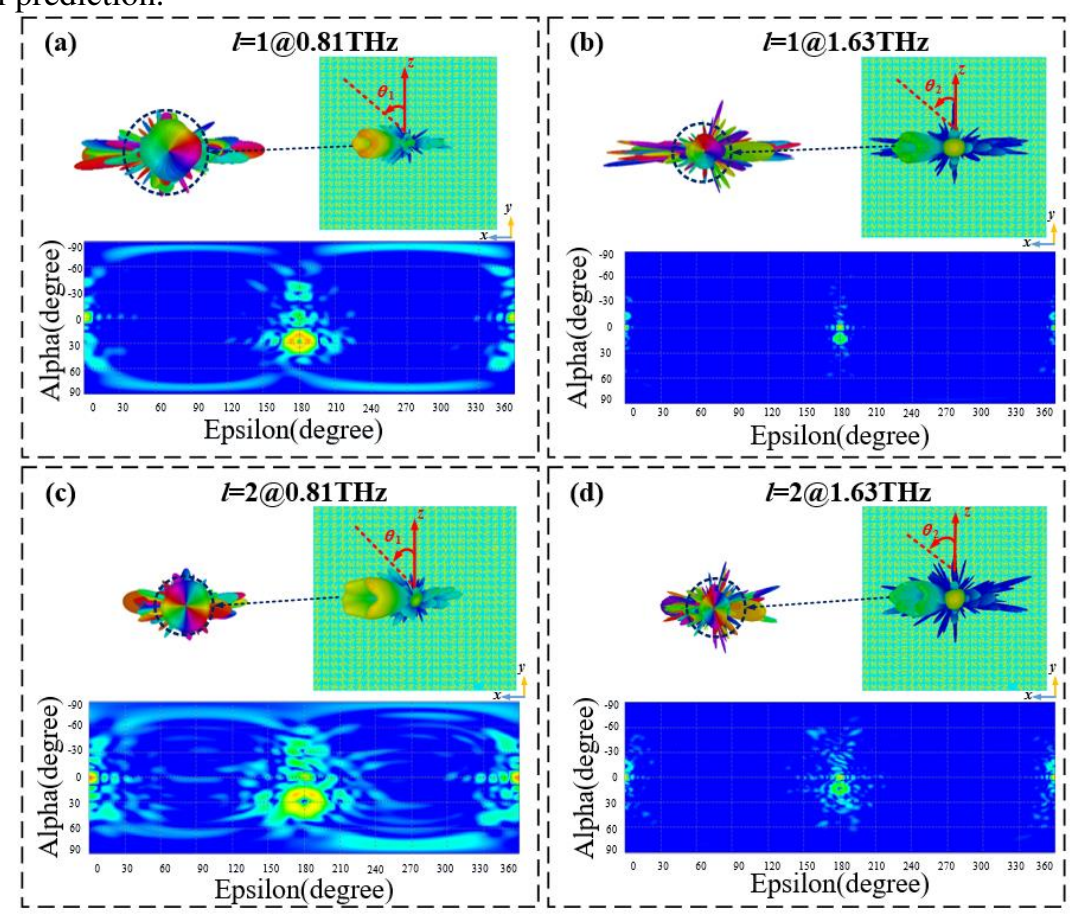

Fig 8. 3D far-field phase patterns, amplitude patterns and $2 \mathrm{D}$ far-field amplitude patterns at (a) $0.81 \mathrm{THz}$ and (b) $1.63 \mathrm{THz}$

\subsection{Dual-frequency transmissive vortex beams generator with symmetric deflection angle}

Finally, a dual-frequency vortex beams generator with two symmetric vortex beams is designed. By adding the gradient phase, the proposed metasurface can deflect different OAM modes vortex beams to different directions. The two vortex beams with OAM mode $l=+1$ and $l=-1$ represent the directions of $+x$ and $-x$, respectively. As illustrated in 
Fig. 9(a), we add the OAM pattern of $l=+1$ into the gradient phase sequence $(01234567 \ldots)$ with a deflection angle along $x$ direction. Similarly, we add the OAM pattern of $l=-1$ into the gradient phase sequence $(7654321 \ldots)$ with a deflection angle along $-x$ direction, as depicted in Fig. 9(b). In order to obtain a function-integrated metasurface for generating dual-vortex beams, we should further do complex addition by (Ding at al. 2019)

$$
\mathrm{e}^{\mathrm{j} \Phi_{1}(x, y)}+\mathrm{e}^{\mathrm{j} \Phi_{2}(x, y)}=\mathrm{A}_{0} \mathrm{e}^{\mathrm{j} \Phi_{0}(x, y)}
$$

where $\Phi_{1}(x, y)$ and $\Phi_{2}(x, y)$ represent the coding phase distributions of two independent functions metasurface (Named $\mathrm{M}_{5}$ and $\mathrm{M}_{6}$ ). Refer to Eq. (10), we can calculate the final phase distribution, which is the superposition of the aforementioned independent functions, as shown in Fig.9(c).



Fig 9 . The calculation process and the final total phase distribution.

The final phase mask pattern $\mathrm{M}_{7}$ is illuminated vertically by LCP (RCP) plane wave. Figures 10(a) and 10(b) display the 3D far-field phase patterns, amplitude patterns and 2D far-field amplitude patterns at $0.81 \mathrm{THz}$ and $1.63 \mathrm{THz}$, respectively. From the phase patterns, it can be found that $2 \pi$ spiral phase of two symmetric beams reveal that the OAM beams having topological charges $l=1$ and $l=-1$. The 3D far-field amplitude patterns directly show that the two vortex beams are steered to different directions as predicted results. It can be observed clearly from 2D far-field amplitude patterns that the deflection angle equals $28^{\circ}$ at $0.81 \mathrm{THz}$ and $13^{\circ}$ at $1.63 \mathrm{THz}$, respectively. Simulation results demonstrate that the proposed metasurface can generate two different OAM vortex beams towards the directions of $+x$ and $-x$ at two frequencies simultaneously.

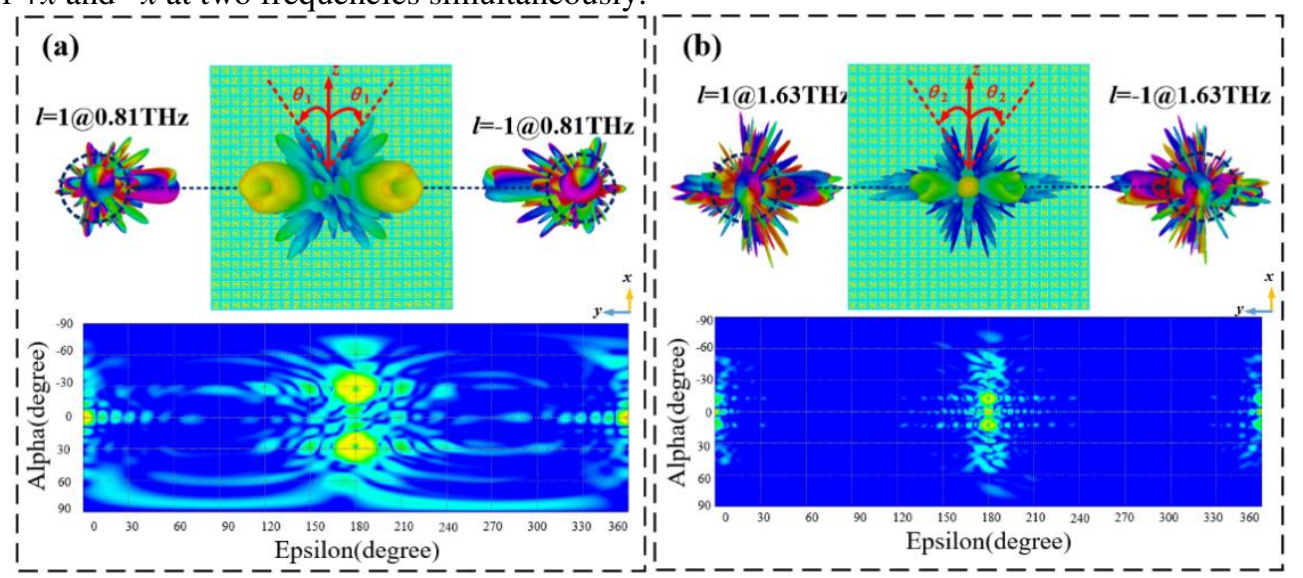

Fig $10.3 \mathrm{D}$ far-field phase, amplitude and $2 \mathrm{D}$ far-field amplitude patterns at (a) $0.81 \mathrm{THz}$ and (b) $1.63 \mathrm{THz}$. 


\section{Conclusion}

To sum up, we proposed a transmissive dual-frequency vortex beams generator by using a novel meta-atom, which is composed of two same metal pattern layers separated by a polymide substrate. The proposed metasurface can generate OAM vortex beams carrying different OAM modes $(l=1$ and $l=2)$ at $0.81 \mathrm{THz}$ and $1.63 \mathrm{THz}$ under circularly polarized (LCP and RCP) incidence. By adding a deflection factor to the metasurface, the OAM vortex beams will be guide to a preset tilted direction. By utilizing complex convolution operation, the proposed metasurface produces two symmetric tilted vortex beams with different OAM modes $(l=+1$ and $l=-1)$ towards the directions of $+x$ and $-x$ at two frequencies. The proposed method provides a convenient and efficient method to generate and control terahertz OAM vortex beams, and has potential applications in terahertz radar and communication.

Acknowledgements This work was supported by the National Natural Science Foundation of China (61871355, 61831012), Zhejiang Key R \& D Project of China under Grant 2021C03153, Fundamental Research Funds for the Provincial Universities of Zhejiang (2020YW20), and Zhejiang Lab (No.2019LC0AB03).

Confict of interest The authors declare no conflicts of interest.

\section{References}

Gibson, G., Courtial, J., Padgett, M., Vasnetsov, M., Pas'ko, V., Barnett, S., and Franke-Arnold, S.: Free-space information transfer using light beams carrying orbital angular momentum. Opt. Express 12(22), 5448-5456(2004).

Chen, H., Hao, J., Zhang, B., Xu, J., Ding, J., and Wang, H.: Generation of vector beam with space-variant distribution of both polarization and phase. Opt. Lett. 36(16), 3179-318(2011).

Allen, L., Beijersbergen, M., Spreeuw, R., Woerdman, J.: Orbital angular momentum of light and transformation of LaguerreGaussian laser modes. Phys. Rev.A, 45(11), 8185-8189(1992).

Tang, S., Li, X., Pan, W., Zhou, J., Jiang, T., Ding, F.: High-efficiency broadband vortex beam generator based on transmissive metasurface. Opt. Express. 27(4), 4281-4291 (2019).

Zhang, X., Kong, D., Yuan, Y., Mei, S., Wang, L., Wang, G.: Broadband and dispersion-free reflective silver metasurfaces as halfwave plate and vortex-beam generator. Opt. Communication. 465,125561 (2020).

Zhang, L., Liu, S., Li, L., and Cui, T.: Spin-Controlled multiple pencil beams and vortex beams with different polarizations generated by Pancharatnam-Berry coding metasurfaces. ACS Appl. Mater. Interfaces. 9(41), 36447-36455(2017).

Akram, M., Bai, X., Jin, R., Vandenbosch, G., and Premaratne, M.: Photon spin hall effect-based ultra-thin transmissive metasurface for efficient generation of OAM waves. IEEE Trans. Antennas Propag. 67(7), 4650-4658(2019).

Qin, F., Wan, L., Li, L., Zhang, H., Wei, G., and Gao, S.: A transmission metasurface for generating OAM beams. IEEE Antennas Wirel. Propag. Lett. 17(10), 1793-1796(2018).

Li, Z., Hao, J., Huang, L., Li, H., Xu, H., Sun, Y., and Dai, N.: Manipulating the wavefront of light by plasmonic metasurfaces operating in high order modes. Opt. Express 24, 8788-8796(2016).

Yang, Y., Yamagami, Y., Yu, X., Pitchappa, P., Webber, J., Zhang, B., Fujita, M., Nagatsuma, T., R. Singh: Terahertz topological photonics for on-chip communication. Nature. Photon.14(7),446-451(2020).

Qi, Q., Chen, X., Zhong, C., Zhang, Z.: Integration of energy, computation and communication in 6G cellular internet of things. IEEE Commun. Lett. 24(6), 1333-1337(2020).

Tang, S., Li, X., Pan, W., Zhou, J., Jiang, T. and Ding, F.: High-efficiency broadband vortex beam generator based on transmissive metasurface. Opt. Express. 27(4), 4281-4291(2019).

Fan. J., Cheng, Y.: Broadband high-efficiency cross-polarization conversion and multi-functional wavefront manipulation based on chiral structure metasurface for terahertz wave. J. Phys. D-Appl. Phys. 53(2), 025109(2020).

Xie, R., Zhai, G., Wang, X., Zhang, D., Si, L., Zhang, H., and Ding, J.: High-efficiency ultrathin dual-wavelength PancharatnamBerry metasurfaces with complete independent phase control. Adv. Opt. Mater. 7(20), 1900594(2019).

Ding, G., Chen, K., Luo, X., Zhao, J., Jiang, T., and Feng, Y.: Dual-Helicity decoupled coding metasurface for independent spinto-orbital angular momentum conversion. Phys. Rev. Appl. 11(4), 044043(2019).

Ran, Y., Liang, J., Cai, T., and Li, H.: High-performance broadband vortex beam generator using reflective Pancharatnam-Berry metasurface. Opt. Commun. 427, 101-106(2018).

Wang, Z., Dong, S., Luo, W., Jia, M., Liang, Z., He, Q., Sun, S., and Zhou, L.: High-efficiency generation of Bessel beams with transmissive metasurfaces. Appl. Phys. Lett. 112(19), 191901(2018).

Ji, C., Song, J., Huang, C., Wu, X., and Luo, X.: Dual-band vortex beam generation with different OAM modes using single-layer metasurface. Opt. Express. 27(1), 34-44(2019).

Xin, M., Xie, R., Zhai, G., Gao, J., Zhang, D., Wang, X., An, S., Zheng, B., Zhang, H., and Ding, J.: Full control of dual-band vortex beams using a high-efficiency single-layer bi-spectral 2-bit coding metasurface. Opt. Express. 28(12), 1737417383(2020).

Wang, T., Xie, R., Zhu, S., Gao, J., Xin, M., An, S., Zheng, B., Li, H., Lin, Y., Zhang, H., Zhuai, G., and Ding, J.: Dual-Band high efficiency terahertz meta-devices based on reflective geometric metasurfaces. IEEE Access 7, 58131-58138(2019). 